Draft version September 16, 2018

Preprint typeset using $\mathrm{LAT}_{\mathrm{E}} \mathrm{X}$ style emulateapj v. 5/2/11

\title{
UPPER BOUNDS ON $r$-MODE AMPLITUDES FROM OBSERVATIONS OF LOW-MASS X-RAY BINARY NEUTRON STARS
}

\author{
Simin Mahmoodifar ${ }^{1}$ and Tod Strohmayer ${ }^{2}$ \\ ${ }^{1}$ Department of Physics, University of Maryland College Park, MD 20742, USA \\ ${ }^{2}$ Astrophysics Science Division, NASA's Goddard Space Flight Center, Greenbelt, MD 20771, USA \\ Draft version September 16, 2018
}

\begin{abstract}
We present upper limits on the amplitude of $r$-mode oscillations, and gravitational-radiation-induced spin-down rates, in low mass X-ray binary neutron stars under the assumption that the quiescent neutron star luminosity is powered by dissipation from a steady-state $r$-mode. For masses $<2 M_{\odot}$ we find dimensionless $r$-mode amplitudes in the range from about $1 \times 10^{-8}$ to $1.5 \times 10^{-6}$. For the accreting millisecond $\mathrm{X}$-ray pulsar sources with known quiescent spin-down rates these limits suggest that $\lesssim 1 \%$ of the observed rate can be due to an unstable $r$-mode. Interestingly, the source with the highest amplitude limit, NGC 6440, could have an $r$-mode spin-down rate comparable to the observed, quiescent rate for SAX J1808-3658. Thus, quiescent spin-down measurements for this source would be particularly interesting. For all sources considered here our amplitude limits suggest that gravitational wave signals are likely too weak for detection with Advanced LIGO. Our highest mass model $\left(2.21 M_{\odot}\right)$ can support enhanced, direct Urca neutrino emission in the core and thus can have higher $r$-mode amplitudes. Indeed, the inferred $r$-mode spin-down rates at these higher amplitudes are inconsistent with the observed spin-down rates for some of the sources, such as IGR J00291+5934 and XTE J1751305. In the absence of other significant sources of internal heat, these results could be used to place an upper limit on the masses of these sources if they were made of hadronic matter, or alternatively it could be used to probe the existence of exotic matter in them if their masses were known.

Subject headings: dense matter - gravitational waves - stars: neutron - stars: oscillations - stars: rotation - X-rays: binaries
\end{abstract}

\section{INTRODUCTION}

The $r$-modes are large-scale currents in neutron stars (NSs) that couple to gravitational radiation and remove energy and angular momentum from the star in the form of gravitational waves (Andersson 1998; Friedman \& Morsink 1998; Friedman \& Schutz 1978; Lindblom et al. 1998). The physics of these oscillations is important in relating the microscopic properties of dense matter-such as its viscosity and neutrino emissivity-to the macroscopic, observable properties of NSs such as their spin frequency, temperature, mass and radius. The $r$-modes are unstable to gravitational radiation and their amplitudes can grow exponentially if viscous and other possible damping mechanisms are not large enough. Because they can affect the dynamic properties of the starits spin frequency and temperature evolution-they are potentially important probes of the phases of dense matter. For example, the boundary of the $r$-mode instability region in the spin frequency - temperature plane, and in particular its minimum, which may determine the final rotation frequency of the star, is very different for stars with different interior compositions (Alford et al. 2012a; Ho et al. 2011; Haskell et al. 2012).

Whether or not the $r$-mode instability limits the spin rates of some NSs it is nevertheless important to explore its potential astrophysically observable signatures. While mass-radius measurements of NSs are important for constraining the equation of state (EOS) of dense matter, observations of their dynamic properties such as spin and thermal evolution are important and potentially more efficient in discriminating between different phases of dense matter. That is because dynamic properties are affected by the transport and thermodynamic properties of dense matter inside the star, such as viscosity, heat conductivity and neutrino emissivity which depend on low energy degrees of freedom and are very different depending on the phase of dense matter present (Alford et al. 2012a, b; Alford \& Schwenzer 2012).

Pulse timing observations of accreting millisecond Xray pulsars (AMXPs) made with NASA's Rossi $X$ ray Timing Explorer ( $R X T E)$ are beginning to reveal the long-term spin evolution of low mass X-ray binary (LMXB) NSs. The pulsar recycling hypothesis whereby millisecond radio pulsars acquire their fast spins via accretion, requires that at least some of these stars are spun-up to hundreds of $\mathrm{Hz}$-the current $\mathrm{NS}$ spin record being $716 \mathrm{~Hz}$ (Hessels et al. 2006). Still, it remains somewhat puzzling that the spin frequency distribution of AMXPs appears to cut-off well below the mass-shedding limit of essentially all realistic NS EOS (Chakrabarty et al. 2003), and it has been suggested that the $r$-mode instability may play a role in limiting NS spin rates (Andersson et al. 1999). Alternatively, recent work supports the idea that at least some of the AMXP population are in or close to "spin equilibrium," essentially determined by the physics of magnetized accretion, and that an additional mechanism, such as the $r$-mode torque, is not required to halt their spin-up (White \& Zhang 1997; Patruno et al. 2012).

While pulse timing noise in AMXPs has made the interpretation of the X-ray pulsation data difficult, there are now several convincing measurements of spin-down during the quiescent phases between accretion outbursts. 
For example, both SAX J1808.4-3658 (hereafter SAX J1808) and IGR J00291+5934 (hereafter IGR J00291) show spin-down of the NS between outbursts, at rates, $\dot{\nu}$, of approximately $1-3 \times 10^{-15} \mathrm{~Hz} \mathrm{~s}^{-1}$ (Patruno 2010; Hartman et al. 2009). The most convincing evidence for an accretion-induced spin-up during outbursts is in IGR J00291, for which a peak value of about $3 \times 10^{-13} \mathrm{~Hz}$ $\mathrm{s}^{-1}$ has now been inferred (Patruno 2010). The magnitude of the "instantaneous" spin-up torque is larger in this source than the spin-down torque by about a factor of 100. The long-term evolution is then a competition between the accretion outbursts that spin it up and the quiescent spin-down intervals. If the outbursts are frequent enough then the star will be spun-up, if not, then long-term spin-down occurs. As the spin-up torque is proportional to the mass accretion rate, the outcome can also be expressed in terms of the long-term, average mass accretion rate.

The observed quiescent spin-down rate, $\dot{\nu}_{s d}$, puts an upper limit on the torque that can be present due to any $r$-mode, as it has to be less than the observed torque of $2 \pi I \dot{\nu}_{s d}$, where $I$ is the moment of inertia of the NS. Now, spin-down of pulsars is typically ascribed to the magnetic-dipole torque that is almost certainly present at some level due to the large scale magnetic field of the NS. Indeed, magnetic field estimates are usually obtained by equating the entire observed spin-down to that expected theoretically for magnetic-dipole radiation, but the observed spin-down is due to the sum total of any torques present. Assuming that both $r$-mode and magnetic-dipole torques are present, then the magnitude of the $r$-mode torque is equal to the observed torque minus the magnetic-dipole torque. Since the magnetic field strengths of AMXPs are typically not known independently from spin-down estimates, the precise value of the magnetic-dipole torque is not known a priori. However, to the extent that the magnetic torque accounts for the majority of the observed spin-down, as is typically assumed, then the $r$-mode torque is likely to be much less than the measured spin-down torque.

In addition to torquing the NS, the viscous damping of the $r$-modes acts as an internal source of heat. Calculations of the coupled thermal and spin evolution of accreting NSs including the effects of $r$-mode heating and gravitational radiation have been carried out by several authors (Brown \& Ushomirsky 2000; Haskell \& Patruno 2011; Ho et al. 2011; Haskell et al. 2012). The primary assumption in these analyses has been that the longterm accretion (spin-up) torque is balanced by the $r$ mode torque due to the emission of gravitational radiation that carries away angular momentum, that is, the long-term average accretion torque is in equilibrium with the $r$-mode torque. As noted above, the recent pulse timing observations of AMXPs indicate that this equilibrium assumption is likely not realized in practice. Moreover, Brown \& Ushomirsky (2000) showed that if the average, fiducial accretion torque given by $N_{a c c}=\langle\dot{M}\rangle(G M R)^{1 / 2}$, where $\langle\dot{M}\rangle$ is the long-term average mass accretion rate, were balanced by the $r$-mode torque then the quiescent luminosities of some accreting NS transients should be substantially larger than observed due to heating from the $r$-modes. Here we use a similar argument to place upper bounds on the $r$-mode amplitudes that can be present in accreting NSs assuming that $r$-mode heating provides the source of NS luminosity in the absence of accretion. We then use these amplitude limits to assess the level of $r$-mode spin-down that can be present, its relation to observed spin-down rates when available, and the expected strength of gravitational radiation.

The paper is organized as follows. In Section 2 we review the basic theory of the $r$-mode instability and how it couples to the thermal and spin evolution of NSs. In Section 3 we describe our methods for constraining the $r$-mode amplitudes using the observed properties of quiescent, LMXB NSs, and we assess the implications for $r$-mode spin-down and the emission of gravitational radiation. We provide a summary and discussion of the implications of our findings in Section 4.

\section{SPIN-DOWN DUE TO UNSTABLE $r$-MODES}

The "flow pattern" of the $r$-modes is prograde in the inertial frame and retrograde in the rotating frame, which means that it moves in the same direction as the star's rotation as seen by an observer at infinity, but in the opposite direction as seen by an observer at rest on the star. Any mode that is retrograde in the co-rotating frame and prograde in the inertial frame grows as a result of its emitting gravitational waves. This is the wellknown Chandrasekhar-Friedman-Schutz mechanism, and it means that gravitational radiation drives the mode rather than dampening it. Viscosity on the other hand tends to damp the $r$-mode by transferring angular momentum from the mode to the rigid (unperturbed) star. The total angular momentum of a perturbed star can be written as

$$
J=I \Omega-J_{c}
$$

where $I$ is the moment of inertia and $\Omega$ is the angular rotation frequency of the star and $J_{c}$ is the canonical angular momentum of the mode, given by

$$
J_{c}=-\frac{3}{2} \tilde{J} M R^{2} \Omega \alpha^{2}
$$

where $\alpha$ is the amplitude of the $r$-mode and $\tilde{J}$ is a dimensionless constant defined by (Owen et al. 1998)

$$
\tilde{J} \equiv \frac{1}{M R^{4}} \int_{0}^{R} \rho r^{6} d r
$$

where $\rho$ is the run of density within the neutron star. The moment of inertia of the star, $I$, can also be written as $I=\tilde{I} M R^{2}$ where $\tilde{I}$ is defined by

$$
\tilde{I} \equiv \frac{8 \pi}{3 M R^{2}} \int_{0}^{R} \rho r^{4} d r .
$$

To derive the equations for the dynamical evolution of the star, we use the following argument by Ho \& Lai (2000). The canonical angular momentum of the mode increases through gravitational radiation and decreases by transferring angular momentum to the star through viscosity

$$
\frac{d J_{c}}{d t}=-\frac{2}{\tau_{G}} J_{c}-\frac{2}{\tau_{V}} J_{c}
$$

where the viscous damping time $\tau_{V}$ is given by $\frac{1}{\tau_{V}}=$ $\frac{1}{\tau_{S}}+\frac{1}{\tau_{B}}+\ldots$. Here, $\tau_{S}$ and $\tau_{B}$ refer to shear and bulk vis- 
cosities, respectively, and the ellipsis denotes other possible dissipative mechanisms, such as boundary layer effects (Wu et al. 2001; Bildsten \& Ushomirsky 2000).

The second evolution equation is obtained by writing the conservation of the total angular momentum $J$ of the perturbed star which says that the total angular momentum of the star decreases due to gravitational radiation and increases due to accretion

$$
\frac{d J}{d t}=-\frac{2 J_{c}}{\tau_{G}}+N_{a c c},
$$

where $N_{a c c}$ is the accretion torque. For a fiducial torque $N_{a c c}$ can be written as $\dot{M}(G M R)^{1 / 2}$ which assumes that each accreted particle transfers to the star an angular momentum equal to the Keplerian value at the stellar radius $R$ (Brown \& Ushomirsky 2000). In the previous equations the quantities $\frac{1}{\tau_{i}}$, where $i$ is either $G, B$, or $S$ for gravitational radiation, bulk viscosity or shear viscosity timescales, respectively, are given by,

$$
\frac{1}{\tau_{i}}=-\frac{P_{i}}{2 E_{c}}
$$

where $E_{c}$ is the canonical energy of the $r$-mode, $P_{G}$ is the power radiated by gravitational waves and $P_{B}$ and $P_{S}$ are the powers dissipated due to bulk and shear viscosity, respectively (in natural units where $c=\hbar=k_{B}=1$ ) (Owen et al. 1998; Alford et al. 2012a),

$$
\begin{aligned}
E_{c} & =\frac{1}{2} \alpha^{2} \Omega^{2} \tilde{J} M R^{2} \\
P_{G} & =\frac{32 \pi(m-1)^{2 m}(m+2)^{2 m+2}}{((2 m+1) ! !)^{2}(m+1)^{2 m+2}} \tilde{J}_{m}^{2} G M^{2} R^{2 m+2} \alpha^{2} \Omega^{2 m+4}
\end{aligned}
$$

$$
\begin{aligned}
P_{B} & =-\frac{16 m}{(2 m+3)(m+1)^{5} \kappa^{2}} \frac{\tilde{V}_{m} \Lambda_{\mathrm{QCD}}^{9-\delta} R^{7} \alpha^{2} \Omega^{4} T^{\delta}}{\Lambda_{E W}^{4}} \\
P_{S} & =-\frac{(m-1)(2 m+1) \tilde{S}_{m} \Lambda_{\mathrm{QCD}}^{3+\sigma} R^{3} \alpha^{2} \Omega^{2}}{T^{\sigma}} .
\end{aligned}
$$

Here we consider $m=2 r$-modes, and $\Lambda_{\mathrm{QCD}}$ and $\Lambda_{\mathrm{EW}}$ are characteristic strong and electroweak scales introduced to make $\tilde{V}$ and $\widetilde{S}$ dimensionless. In our calculations we have used $\Lambda_{Q C D}=1 \mathrm{GeV}$ and $\Lambda_{E W}=100 \mathrm{GeV}$. The dimensionless parameters $\tilde{V}, \tilde{S}, \tilde{I}$ and $\tilde{J}$, which involve radial integration over the star, and $\delta$ and $\sigma$ are given in Table 1 for three different NS models that we study in this paper. All three models here are made of non-superfluid, hadronic npe matter with the APR EOS (Akmal et al. 1998), which generates a reasonable NS mass-radius relation that is consistent with observational constraints (labeled AP4 in Hebeler et al. (2013) and Lattimer (2012)), but they have different masses $\left(1.4 M_{\odot}, 2.0 M_{\odot}\right.$ and $\left.2.21 M_{\odot}\right)$ and radii (Alford et al. 2012a). The two models with masses of $1.4 M_{\odot}$ and $2.0 M_{\odot}$ only allow modified Urca neutrino emission in the core, but the one with a mass of $2.21 M_{\odot}$ allows direct Urca neutrino emission in a core of radius $5.9 \mathrm{~km}$. Direct Urca processes are very sensitive to the proton fraction of dense matter. The required proton fraction is roughly $14 \%$ in the case of the APR EOS, reached at relatively high density $n \sim 5 n_{0}$, where $n_{0}$ is the nuclear saturation density, this could be different for other EOSs (Alford et al. 2012a).

The evolution equations for the amplitude of the $r$ mode, $\alpha$, and spin frequency of the star, $\Omega$, can be written by using Equations 5 and $\left[6\right.$ and substituting $J_{c}$ from Equation 2. The third equation -which describes the temperature evolution- can be obtained by noting that the temperature of the star decreases due to thermal emission from the surface and neutrino emission from the interior, which in an average mass hadronic star is dominated by modified Urca processes (in a massive star direct Urca processes can also occur in the core and should be included in the neutrino emissivity as well), and it increases due to the viscous dissipation of the $r$-mode energy, $P_{V}$. This gives the following equations for the evolution of spin frequency, $r$-mode amplitude and temperature,

$$
\begin{aligned}
& \frac{d \Omega}{d t}=-2 Q \frac{\Omega \alpha^{2}}{\tau_{V}}+\frac{N_{a c c}}{I} \\
& \frac{d \alpha}{d t}=-\frac{\alpha}{\tau_{G}}-\frac{\alpha}{\tau_{V}}\left(1-\alpha^{2} Q\right)-\frac{\alpha}{2 \Omega} \frac{N_{a c c}}{I} \\
& \frac{d E}{d t}=C_{V} \frac{d T}{d t}=-L_{\nu}-L_{\gamma}+\left|P_{V}\right|+H,
\end{aligned}
$$

where $Q \equiv \frac{3 \tilde{J}}{2 \tilde{I}}$, and the viscous dissipated power is $P_{V}=P_{S}+P_{B}+\ldots$, and again the ellipsis denotes other possible dissipative processes. In Equation (12c), $H$ represents other heating mechanisms that might be present in the star, but are not related to the $r$-mode dissipation, such as deep crustal heating due to nuclear reactions in the NS crust Haensel \& Zdunik 2003; Gupta et al. 2007). Since our goal in this paper is to obtain upper limits on $r$-mode amplitudes we can safely set $H=0$. We discuss this further in the next section. Here, $L_{\nu}, L_{\gamma}$ and $C_{V}$, which are the total neutrino luminosity, photon luminosity and specific heat of the star, are given by (in natural units)

$$
\begin{aligned}
L_{\nu} & =\frac{4 \pi R^{3} \Lambda_{Q C D}^{9-\theta} \tilde{L}}{\Lambda_{E W}^{4}} T^{\theta} \\
L_{\gamma} & =4 \pi R^{2} \sigma T_{e f f}^{4} \\
C_{V} & =4 \pi \Lambda_{Q C D}^{3-v} R^{3} \tilde{C}_{V} T^{v},
\end{aligned}
$$

where $T$ is the core temperature of the star and $T_{\text {eff }}$ is the surface temperature. The dimensionless parameters $\tilde{L}_{\nu}$ and $\tilde{C}_{V}$ (that involve radial integration over the star (Alford \& Schwenzer 2012)) and $\theta$ and $v$ are given in Table 1 for different stellar models.

Figure 11 shows the $r$-mode instability window computed for a $1.4 M_{\odot}$ NS using the APR EOS (the modification to the instability window is rather modest in the case of the two other stellar models considered in this paper (Alford et al. 2012a)). In the region above the curve the gravitational radiation timescale is smaller than the viscous damping timescales, therefore $r$-modes are unstable and their amplitudes grow exponentially in this region. As can be seen in this figure most of the LMXB's are in the unstable region for normal hadronic stars where the damping is due to shear viscosity from 
TABLE 1

Parameters of the Neutron Star Models

\begin{tabular}{|c|c|c|c|c|c|c|c|c|c|c|c|c|c|}
\hline Neutron Star & Shell & $R(\mathrm{~km})$ & $\Omega_{K}(\mathrm{~Hz})$ & $\tilde{I}$ & $\tilde{J}$ & $\tilde{S}$ & $\tilde{V}$ & $\tilde{C}_{V}$ & $\tilde{L}$ & $\sigma$ & $\delta$ & $v$ & $\theta$ \\
\hline NS $1.4 M_{\odot}$ & Core & 11.5 & 6020 & 0.283 & $1.81 \times 10^{-2}$ & $7.68 \times 10^{-5}$ & $1.31 \times 10^{-3}$ & $2.36 \times 10^{-2}$ & $1.91 \times 10^{-2}$ & $\frac{5}{3}$ & 6 & 1 & 8 \\
\hline $\mathrm{NS} 2.0 M_{\odot}$ & Core & 11.0 & 7670 & 0.300 & $2.05 \times 10^{-2}$ & $2.25 \times 10^{-4}$ & $1.16 \times 10^{-3}$ & $2.64 \times 10^{-2}$ & $1.69 \times 10^{-2}$ & $\prime \prime$ & $\prime \prime$ & $\prime \prime$ & $\prime \prime$ \\
\hline \multirow[t]{2}{*}{$\mathrm{NS} 2.21 M_{\odot}$} & m.U. core & 10.0 & 9310 & 0.295 & $2.02 \times 10^{-2}$ & $5.05 \times 10^{-4}$ & $9.34 \times 10^{-4}$ & $2.62 \times 10^{-2}$ & $1.29 \times 10^{-2}$ & $\prime \prime$ & $\prime \prime$ & $\prime \prime$ & "1 \\
\hline & d.U. core & & & & & & $1.16 \times 10^{-8}$ & & $2.31 \times 10^{-5}$ & & 4 & & 6 \\
\hline
\end{tabular}

Note. - Radius, Kepler frequency and radial integral parameters that appear in the moment of inertia, angular momentum of the mode, dissipative powers due to shear viscosity (from leptonic interactions) and bulk viscosity (due to Urca processes), specific heat and neutrino luminosity for different NS models considered in this work (Alford \& Schwenzer 2012; Alford et al. 2012a). For the 2.21 $M_{\odot}$ model the bulk viscosity and neutrino luminosity parameters are different in the inner core where direct Urca processes are allowed, therefore these values are given separately in the last row.

leptonic scattering (i.e. electron-electron and electronproton scatterings, which are the dominant contributions to the shear viscosity of normal hadronic matter in NS cores) and bulk viscosity due to Urca processes, therefore, there must be some non-linear mechanism that saturates the amplitude of the unstable $r$-modes at a finite value. Supra-thermal bulk viscosity (Alford et al. 2010) is one of these non-linear mechanisms, but in the case that only the core of the star is considered and the effects of the crust are ignored, it can only saturate the $r$ mode at large amplitudes, $(\alpha \sim 1)$ (Alford et al. 2012c). Magnetohydrodynamic coupling to the stellar magnetic field is another mechanism that can damp the $r$-mode instability, but it can only saturate the $r$-mode at large amplitudes $(\alpha \gtrsim 0.01)$ in the presence of a magnetic field significantly larger than $\sim 10^{8} \mathrm{G}$ that is characteristic of the LMXB sources considered here (Rezzolla et al. 2000, 2001). Mode coupling can saturate the $r$-mode at smaller amplitudes $\left(\alpha \sim 10^{-4}\right)$, but those values are still very large compared to the upper limits we find in this work (Arras et al. 2003; Bondarescu et al. 2007, 2009). At this point it is not entirely clear which mechanism is actually responsible for saturating the $r$-mode amplitude (none of the saturation mechanisms proposed so far can saturate $r$-modes at the low amplitudes we find here), however, in this paper our primary interest is to obtain upper bounds on $r$-mode amplitudes from observations of NS transients and this does not require a precise understanding of the saturation mechanism. Understanding the detailed physics of the saturation mechanism is an important issue, but it is beyond the scope of this paper.

Here we note that if we were to consider the existence of exotic forms of matter, such as strange quark matter in the star, then the shape of the instability window would change due to their different shear and bulk viscosities, and it is possible that the LMXBs considered here might fall outside of the resulting instability window Alford et al. 2012a; Haskell et al. 2012; Schwenzer 2012). The shape of the instability window may also be different due to crust boundary layer effects (Ho et al. 2011), but even in that case for realistic boundary layer models most of the LMXB sources are in the unstable region. Therefore, we assume that these sources have unstable $r$-modes which are emitting gravitational waves but the amplitude of the $r$-mode is not growing exponentially, and there is a mechanism that can saturate the growth of the $r$-mode. In addition, we assume that all of these stars are made of normal hadronic matter (constructed with the APR EOS) and all of the sources that are in the unstable region for hadronic stars in Figure 1

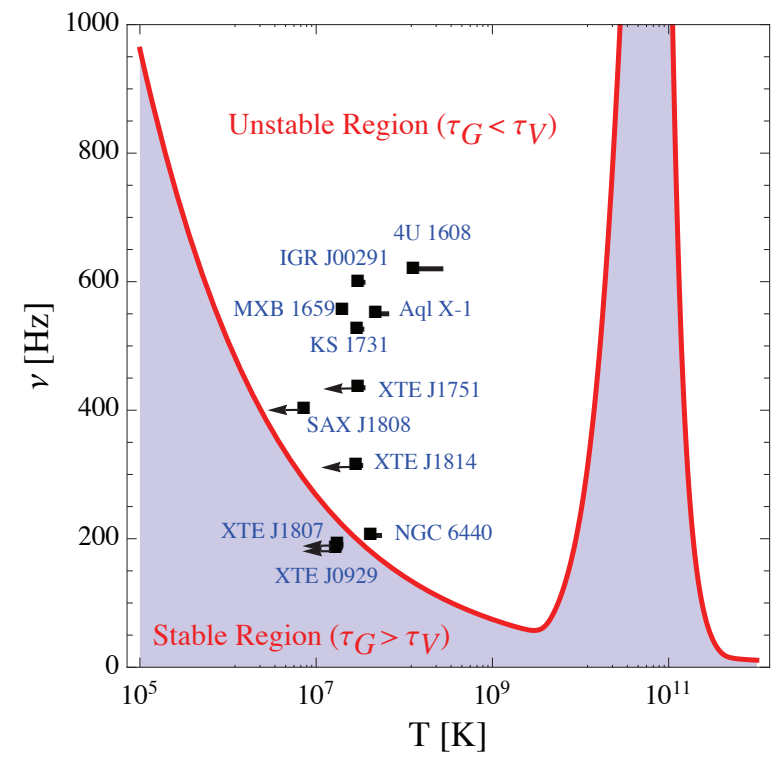

FIG. 1. - The $r$-mode instability region for a $1.4 M_{\odot} \mathrm{NS}$ constructed with the APR EOS in the spin frequency vs. core temperature plane. Also shown are some of the LMXBs which have been considered in this work. The horizontal line extending rightward from the temperature symbols (the black squares) shows the difference between two models for relating the surface temperature to the core temperature (i.e. the difference between a fully or partially accreted envelope). The difference between the core temperatures in these two cases gets larger as the surface temperature increases, but even for the highest surface temperature considered in this work the difference is not large enough to change our results.

(A color version of this figure is available in the online journal.)

are emitting gravitational waves at a constant amplitude.

Since we do not know which mechanism is actually responsible for saturating the $r$-mode amplitude, we simply assume that there is a nonlinear mechanism that saturates the mode. When $\alpha$ hits the saturation amplitude, the right hand side of Equation (12b) becomes zero which implies that

$$
\frac{1}{\tau_{V}}=\frac{1}{\tau_{G}} \frac{1}{1-\alpha^{2} Q},
$$

where we have neglected the last term in Equation (12b) since it is much smaller than the other terms.

Therefore, when the amplitude is saturated, in all of the evolution equations $\frac{1}{\tau_{V}}$ should be replaced by $\frac{1}{\tau_{G}}\left(\frac{1}{1-\alpha^{2} Q}\right)$ and $\left|P_{V}\right|$ by $\frac{P_{G}}{1-\alpha^{2} Q}$. Since viscosity alone cannot stop the growth of the $r$-mode and an extra mechanism is needed to do that, therefore at saturation $\frac{1}{\tau_{G}}\left(\frac{1}{1-\alpha^{2} Q}\right)$ is larger than $\frac{1}{\tau_{V}}$ and the reheating term on 
TABLE 2

Constraints from Spin Equilibrium

\begin{tabular}{crrrrr}
\hline \hline Source & $\Delta=\frac{t_{o}}{t_{r}}$ & $\begin{array}{r}\dot{\nu}_{\text {su }} \\
\left(\mathrm{Hz} \mathrm{s}^{-1}\right)\end{array}$ & $\begin{array}{r}\alpha_{\text {sp.eq }} \\
\left(1.4 M_{\odot}\right)\end{array}$ & $\begin{array}{r}\alpha_{\text {sp.eq }} \\
\left(2.0 M_{\odot}\right)\end{array}$ & $\begin{array}{r}\alpha_{\text {sp.eq }} \\
\left(2.21 M_{\odot}\right)\end{array}$ \\
\hline IGR J00291 & $\frac{13}{1363}$ & $5 \times 10^{-13}$ & $1.46 \times 10^{-7}$ & $1.22 \times 10^{-7}$ & $1.41 \times 10^{-7}$ \\
SAX J1808-3658 & $\frac{40}{2 \times 365}$ & $2.5 \times 10^{-14}$ & $3.20 \times 10^{-7}$ & $2.66 \times 10^{-7}$ & $3.08 \times 10^{-7}$ \\
XTE J1814-338 & $\frac{50}{19 \times 365}$ & $1.5 \times 10^{-14}$ & $2.12 \times 10^{-7}$ & $1.76 \times 10^{-7}$ & $2.04 \times 10^{-7}$ \\
\hline
\end{tabular}

Note. - Duty cycle $\Delta$, spin-up rate $\dot{\nu}_{s u}$ (Patruno \& Watts 2012), and upper limits on $r$-mode amplitudes from the "spinequilibrium" condition $\alpha_{s p . e q}$, for three neutron star transients with assumed masses of $1.4 M_{\odot}, 2.0 M_{\odot}$ and $2.21 M_{\odot}$.

the right hand side of the temperature evolution equation (Eq 12C) will be larger than the reheating due to bulk and shear viscosity (Alford et al. 2012b).

\section{CONSTRAINING THE $r$-MODE AMPLITUDE}

\subsection{Constraints from "Spin Equilibrium"}

Here we compare two different methods for constraining the $r$-mode amplitude, $\alpha$, from observations of LMXB NS transients. The first one, which gives larger values for $\alpha$, is based on the spin equilibrium assumption (Brown \& Ushomirskv 2000; Ho et al. 2011; Haskell \& Patruno |2011; Watts et al. 2008) where we assume that in an outburst-quiescence cycle all the spinup torque due to accretion during the outburst is balanced by the $r$-mode spin-down torque due to gravitational radiation in the whole cycle. This is similar to the prescription considered by previous authors (Brown \& Ushomirskv 2000; Ho et al. 2011), but rather than using a "fiducial" torque estimated from the longterm average $\dot{M}$, we can now use the observed spin-up rates and outburst properties to directly constrain the torque. Therefore we have

$$
2 \pi I \dot{\nu} \Delta=\frac{2 J_{c}}{\tau_{G}}
$$

where $\dot{\nu}$ is the spin-up rate during outburst and $\Delta=\frac{t_{o}}{t_{r}}$ is the ratio of the outburst duration, $t_{o}$, to the recurrence time, $t_{r}$. We can estimate the left hand side of Equation (17) from X-ray observations of LMXBs and since the right hand side is a function of $\alpha$ through $J_{c}$ (see Equation (2)) we can determine $\alpha$. In Table 2 we give the results for $\alpha$ for three sources for which there are now estimates of the spin-up rate due to accretion. The values of $\alpha$ are given for the three different NS models considered in this work, and they are all in the range of $\approx 1-3 \times 10^{-7}$.

At these amplitudes the inferred $r$-mode spin-down rate would be competitive with the magnetic dipole spin-down rate which almost certainly exists in these LMXBs, and which is quite likely the dominant spindown mechanism. Moreover, the amplitudes are also comparable to those deduced assuming $r$-mode spin equilibrium with the "fiducial" accretion torques estimated by Brown \& Ushomirsky (2000). Those authors also demonstrated that at such amplitudes some of these objects should have significantly higher quiescent luminosities due to $r$-mode reheating than observed. These results suggest that the $r$-mode torque does not balance the accretion-driven spin-up torque and that $r$-mode amplitude estimates based on the "spin equilibrium" assumption will overestimate the true amplitude.

\subsection{Constraints from "Thermal Equilibrium"}

Here, we use the same thermal equilibrium argument outlined by Brown \& Ushomirsky, but rather than estimating the quiescent luminosity using the $r$-mode amplitude deduced from "spin equilibrium," we use observations of the quiescent luminosity of LMXBs to directly constrain the amplitude of the $r$-mode. This works because in a steady-state, gravitational radiation pumps energy into the $r$-mode at a rate given by, $W_{d}=(1 / 3) \Omega \dot{J}_{c}=$ $-2 E_{c} / \tau_{G}$. This expression has the familiar relationship for a power dissipated by an applied torque, and in this case it is simply the $r$-mode torque due to gravitational radiation. In a thermal steady-state all of this energy must be dissipated in the star. Some fraction of this heat will be lost from the star due to neutrino emission and the rest will be radiated at the surface. It should be mentioned that the thermal steady-state is not an assumption, but a rigorous result when the mode is saturated, and in particular it is independent of the cooling mechanism (Alford \& Schwenzer 2012). We further assume that all of the energy emitted from the star during quiescence is due to the $r$-mode dissipation inside the star. This is equivalent to setting $H=0$ in Equation (12c). The resulting $r$-mode amplitude limits are upper bounds in the sense that the observed luminosity reflects the contribution from $r$-mode heating as well as any additional sources of heat that are present, such as for example due to accretion and the nuclear processing of accreted material, so-called deep crustal heating (Haensel \& Zdunik 2003; Gupta et al. 2007). If any such sources of heat are present then the actual $r$-mode amplitude will be less than the upper bounds given here. For the sources that we study in this work, since we know the values of surface temperatures and quiescent luminosities from observations, we can estimate the core temperature and therefore determine the neutrino luminosities to estimate the total amount of heat deposited in the core of these systems by gravitational radiation.

To compute the core temperatures we use equation A8 in Potekhin et al. (1997) which relates the effective surface temperature of the star $T_{\text {eff }}$ to the internal temperature $T_{b}$, which is the temperature at a fiducial boundary at $\rho_{b}=10^{10} \mathrm{~g} \mathrm{~cm}^{-3}$ for a fully accreted envelope and is valid at not too high temperatures $\left(T_{b} \leq 10^{8} K\right)$,

$$
\left(\frac{T_{\text {eff }}}{10^{6} \mathrm{~K}}\right)^{4}=\left(\frac{g}{10^{14} \mathrm{cms}^{-2}}\right)\left(18.1 \frac{T_{b}}{10^{9} \mathrm{~K}}\right)^{2.42}
$$

where $g=G M /\left(R^{2} \sqrt{1-r_{g} / R}\right)$ is the surface gravity and $r_{g}=2 G M / c^{2}$. Here we assume that the NS's core is isothermal and since the thermal conductivity of the crust is high (Brown \& Cumming 2009) we have $T_{\text {core }}=T_{b}$ to good approximation. If we instead use equation A9 in Potekhin et al. (1997) which gives $T_{b}$ for a partially accreted envelope, and a column depth of $\frac{P}{g}=10^{9} \mathrm{~g} \mathrm{~cm}^{-2}$ (Haskell et al. 2012) we get core temperatures slightly higher than those derived from Equation (18). The right hand side of the error bars on the temperatures in Figure 1 shows this difference. It is really only relevant for a single source, $4 \mathrm{U} 1608-522$, but even in this case it is less than a $50 \%$ increase, and the difference is always small enough that it doesn't qualitatively change our results in the remainder of the paper. To compute 
TABLE 3

Estimates of NS Temperatures and Luminosities

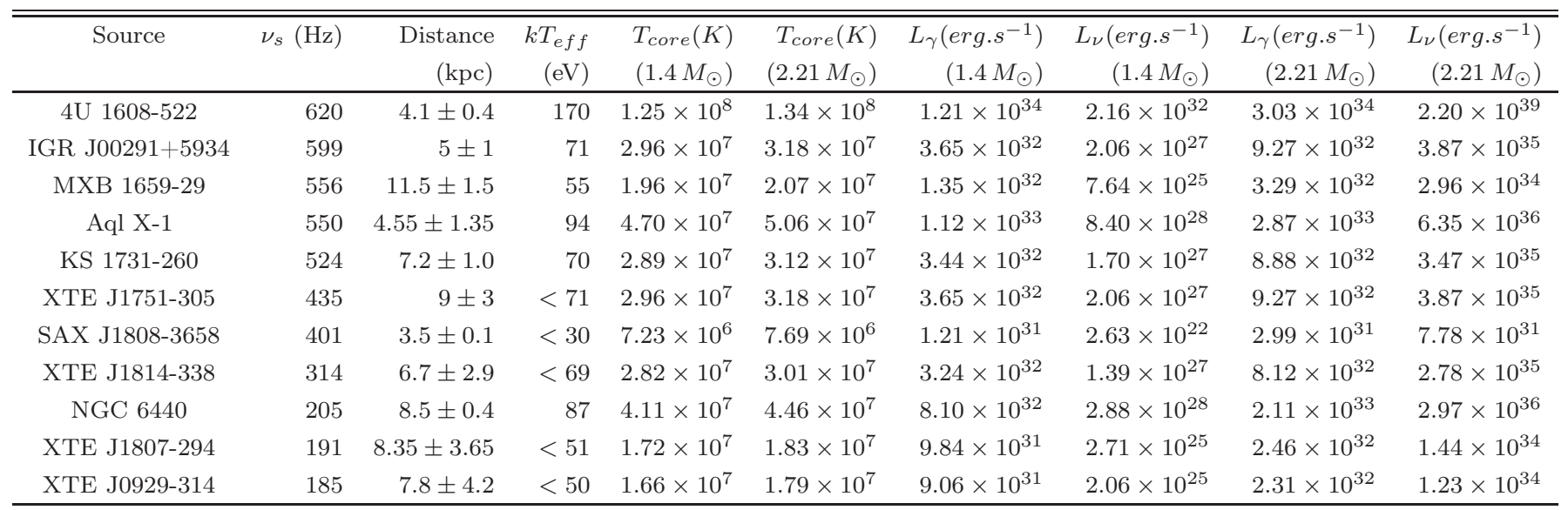

Note. - Spin frequency, distance to the source (Watts et al. 2008), effective temperature at the surface of the star (Heinke et al. 2007, 2009; Tomsick et al. 2004), core temperature, photon luminosity at the surface of the star and neutrino luminosity for both $1.4 M_{\odot}$ and $2.21 M_{\odot}$ NSs. Note that $k T_{\text {eff }}$ given in this table is for a $1.4 M_{\odot}$ NS with a radius of $10 \mathrm{~km}$ but in computing the core temperatures and luminosities for different NS models the appropriate redshifts have been used. We note that for the sake of brevity the core temperatures for the $2 M_{\odot}$ models are not included in the table; however, their values are all $\approx 5 \%$ less than those for the $2.21 M_{\odot}$ model.

$T_{b}$ we have used the effective surface temperatures, $T_{e f f}$, given by Heinke et al. (2007, 2009) and Tomsick et al. (2004)]. Note that those temperatures are computed for a $1.4 M_{\odot}$ NS with a $10 \mathrm{~km}$ radius and we have used the appropriate redshifts to compute $T_{\text {core }}$ for our neutron star models.

Having surface and core temperatures for these sources we can use Equations (13) and (14) to evaluate their neutrino and thermal luminosities for different stellar models. The values of the core temperatures, as well as the photon and neutrino luminosities for different sources for the $1.4 M_{\odot}$ and $2.21 M_{\odot}$ NS models are computed and given in Table 3 . Note that in the case of $1.4 M_{\odot}$ NSs the neutrino luminosity is only due to the modified Urca reactions, but for the $2.21 M_{\odot} \mathrm{NS}$ it is due to both direct and modified Urca reactions. By comparing the neutrino and thermal luminosities in Table 3 one can see that at temperatures relevant for the LMXBs the standard neutrino cooling from modified Urca reactions is negligible compared to the photon emission from the surface of the star. However, if the star is massive enough to enable direct Urca reactions in the core (such as for our $2.21 M_{\odot}$ NS model) then the neutrino emission will dominate the cooling process for surface temperatures higher than about $34 \mathrm{eV}$ (Brown et al. 1998).

The thermal equilibrium condition can be written as $W_{d}=L_{\nu}+L_{\gamma}$, where reheating due to $r$-mode dissipation is given by $W_{d}=\frac{-2 E_{c}}{\tau_{G R}}$ and is a function of $r$-mode amplitude, $\alpha$. Therefore, $\alpha$ can be written in terms of luminosities as

$$
\alpha=\frac{5 \times 3^{4}}{2^{8} \tilde{J} M R^{3} \Omega^{4}}\left(\frac{L_{\gamma}+L_{\nu}}{2 \pi G}\right)^{1 / 2}
$$

where $L_{\gamma}=4 \pi R^{2} \sigma T_{e f f}^{4}$ is the thermal photon luminosity

1 It should be emphasized that the temperatures given in Table 2 of Heinke et al. (2007, 2009) are effective temperatures at the surface of the star and not redshifted surface temperatures seen by an observer at infinity. We note that they have been incorrectly assumed to be redshifted temperatures in Degenaar et al. (2012) and Haskell et al. (2012). at the surface of the star. Here, $R$ and $T_{\text {eff }}$ are the stellar radius and surface temperature, respectively, and the neutrino luminosity is given by

$$
L_{\nu}=\frac{4 \pi R^{3} \Lambda_{Q C D}^{3} \tilde{L}_{D U}}{\Lambda_{E W}^{4}} T^{6}+\frac{4 \pi R^{3} \Lambda_{Q C D} \tilde{L}_{M U}}{\Lambda_{E W}^{4}} T^{8}
$$

where $T$ is the core temperature, $R_{D U}$ is the radius of the core where direct Urca neutrino emission is allowed and $\tilde{L}$ is a dimensionless parameter given in Table 1] The thermal equilibrium condition for an NS with standard neutrino cooling $\left(1.4 M_{\odot}\right.$ and $2.0 M_{\odot}$ NSs in this study) can be approximated as $W_{d} \simeq L_{\gamma}$, since the neutrino cooling in this case is negligible compared to the surface photon luminosity.

As can be seen in Figure 1 out of the 11 sources considered in this paper all but 2 of them are likely to have unstable $r$-modes, meaning that they are above the $r$-mode instability curve. The two most slowly rotating sources, XTE J1807 and XTE J0929, are outside the instability region for our NS models, which means they likely can no longer spin-down due to gravitational radiation from an $r$-mode2. Therefore, we only evaluate the upper bounds on the $r$-mode amplitude for those nine sources within the instability window. Using Equation (19), we have evaluated $\alpha$ for all of those sources using the three different NS models considered in this work. The values of $\alpha$ are given in Table 4 As can be seen for the $1.4 M_{\odot}$ and 2.0 $M_{\odot}$ NSs, where there is no enhanced neutrino emission, the values of $\alpha$ range from $1.07 \times 10^{-8}$ to $1.54 \times 10^{-6}$, where NGC 6440-which has the lowest spin frequencyhas the highest $r$-mode amplitude, as expected. In the case of $2.21 M_{\odot}$ NSs the upper bounds on $\alpha$ are larger since due to the direct Urca neutrino emission $W_{d}$ can be larger, and $\alpha$ in this case ranges from $3.30 \times 10^{-8}$ to $8.03 \times 10^{-5}$. $4 \mathrm{U} 1608-522$ has the highest temperature among the sources considered which implies a very large

2 It has been shown by Alford et al. (2012a) that the boundary of the instability region is insensitive to the quantitative details of the microscopic interactions that induce viscous damping in a given phase of dense matter. 
TABLE 4

Upper Bounds on $r$-mode Amplitudes and NS Spin-down Rates

\begin{tabular}{|c|c|c|c|c|c|c|c|}
\hline Source & $\begin{array}{r}\alpha_{t h . e q} \\
\left(1.4 M_{\odot}\right) \\
\end{array}$ & $\begin{array}{r}\alpha_{t h . e q} \\
\left(2.0 M_{\odot}\right) \\
\end{array}$ & $\begin{array}{r}\alpha_{\text {th.eq }} \\
\left(2.21 M_{\odot}\right) \\
\end{array}$ & $\begin{array}{r}\dot{\nu}\left(\mathrm{Hz} \mathrm{s}^{-1}\right) \\
\left(1.4 M_{\odot}\right)\end{array}$ & $\begin{array}{r}\dot{\nu}\left(\mathrm{Hz} \mathrm{s}^{-1}\right) \\
\left(2.0 M_{\odot}\right)\end{array}$ & $\begin{array}{r}\dot{\nu}\left(\mathrm{Hz} \mathrm{s}^{-1}\right) \\
\left(2.21 M_{\odot}\right)\end{array}$ & $\begin{array}{r}\dot{\nu}_{s d}\left(\mathrm{~Hz} \mathrm{~s}^{-1}\right) \\
\text { observation }\end{array}$ \\
\hline $4 \mathrm{U} 1608-522$ & $7.15 \times 10^{-8}$ & $6.60 \times 10^{-8}$ & $2.61 \times 10^{-5}$ & $-1.44 \times 10^{-15}$ & $-1.78 \times 10^{-15}$ & $-2.08 \times 10^{-10}$ & \\
\hline IGR J00291+5934 & $1.41 \times 10^{-8}$ & $1.32 \times 10^{-8}$ & $3.99 \times 10^{-7}$ & $-4.42 \times 10^{-17}$ & $-5.59 \times 10^{-17}$ & $-3.82 \times 10^{-14}$ & $-3 \times 10^{-15}$ \\
\hline MXB 1659-29 & $1.16 \times 10^{-8}$ & $1.07 \times 10^{-8}$ & $1.49 \times 10^{-7}$ & $-1.78 \times 10^{-17}$ & $-2.18 \times 10^{-17}$ & $-3.16 \times 10^{-15}$ & \\
\hline Aql X-1 & $3.49 \times 10^{-8}$ & $3.27 \times 10^{-8}$ & $2.26 \times 10^{-6}$ & $-1.49 \times 10^{-16}$ & $-1.89 \times 10^{-16}$ & $-6.74 \times 10^{-13}$ & \\
\hline KS 1731-260 & $2.35 \times 10^{-8}$ & $2.20 \times 10^{-8}$ & $6.44 \times 10^{-7}$ & $-4.81 \times 10^{-17}$ & $-6.09 \times 10^{-17}$ & $-3.90 \times 10^{-14}$ & \\
\hline XTE J1751-305 & $5.09 \times 10^{-8}$ & $4.76 \times 10^{-8}$ & $1.44 \times 10^{-6}$ & $-6.13 \times 10^{-17}$ & $-7.74 \times 10^{-17}$ & $-5.29 \times 10^{-14}$ & $-5.5 \times 10^{-15}$ \\
\hline SAX J1808-3658 & $1.28 \times 10^{-8}$ & $1.19 \times 10^{-8}$ & $3.30 \times 10^{-8}$ & $-2.19 \times 10^{-18}$ & $-2.74 \times 10^{-18}$ & $-1.57 \times 10^{-17}$ & $-5.5 \times 10^{-16}$ \\
\hline XTE J1814-338 & $1.76 \times 10^{-7}$ & $1.67 \times 10^{-7}$ & $4.49 \times 10^{-6}$ & $-7.49 \times 10^{-17}$ & $-9.73 \times 10^{-17}$ & $-5.26 \times 10^{-14}$ & \\
\hline NGC 6440 & $1.54 \times 10^{-6}$ & $1.45 \times 10^{-6}$ & $8.03 \times 10^{-5}$ & $-2.90 \times 10^{-16}$ & $-3.71 \times 10^{-16}$ & $-8.50 \times 10^{-13}$ & \\
\hline
\end{tabular}

Nоте. - Upper bounds on the $r$-mode amplitude from the "thermal equilibrium" condition that are consistent with quiescent luminosity data are given for different neutron star models. The gravitational radiation induced spin-down rates due to unstable $r$-modes as well as the observed spin-down rate for some of the sources are also given (Patruno 2010; (Patruno \& Watts 2012; (Patruno et al. 2011).

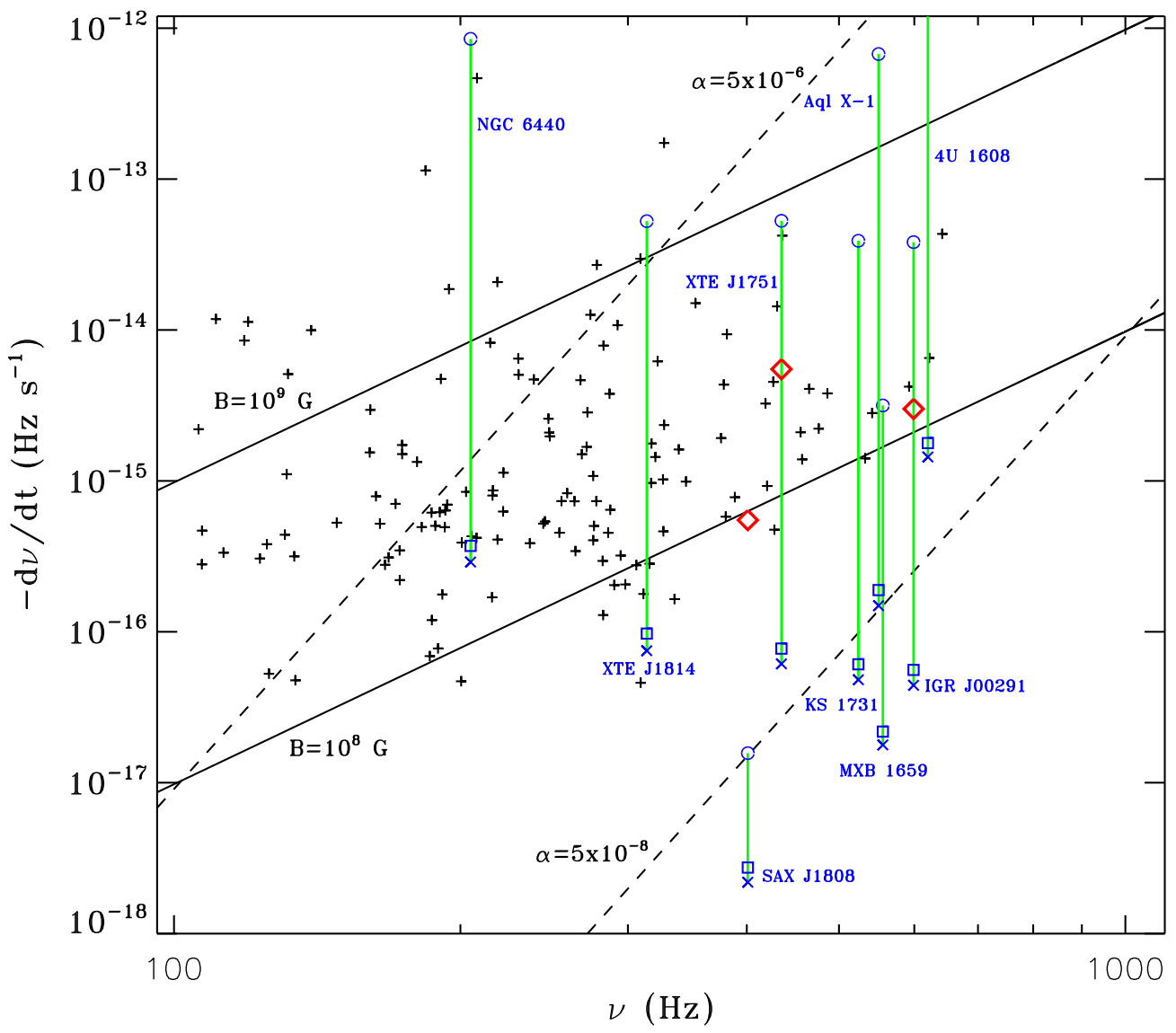

FIG. 2.- Limits on the spin-down rates due to an $r$-mode torque for nine LMXB systems and a range of NS masses are shown in the $\dot{\nu}$ vs. $\nu$ plane. The $\dot{\nu}$ limits for $1.4,2.0$, and $2.21 M_{\odot}$ NS models obtained from the $r$-mode amplitude limits derived from observations of quiescent luminosities and temperatures (see the discussion in Section 3) are marked by the $\times$, square, and circle symbols, respectively. The vertical green lines connecting the symbols show the full range of $\dot{\nu}$ for each source (labeled). Also shown are two pairs of parallel lines representing magnetic-dipole (solid) and $r$-mode (dashed) braking laws. Lines are drawn for two values of the magnetic field, $10^{8} \mathrm{G}$ (lower), and $10^{9} \mathrm{G}$ (upper), as well as two values of $\alpha, 5 \times 10^{-8}$ (lower) and $5 \times 10^{-6}$ (upper). For systems with measured, quiescent spin-down rates, these values are marked with the red diamond symbols. For additional context, millisecond pulsars from the ATNF pulsar database (Manchester et al. 2005) are shown and denoted by the black + symbols.

(A color version of this figure is available in the online journal.) 
neutrino luminosity for the $2.21 M_{\odot}$ NS model and as a result a large $r$-mode amplitude. The large value of the $r$-mode amplitude in this source may be ruled out by measurement of its spin-down rate, as is explained further in the next section. The high temperature of $4 \mathrm{U}$ 1608-522 could be explained if it has a lower mass, but it should also be noted that this system has been accreting for a long time and both its high quiescent luminosity and surface temperature may be due to the long term accretion and our assumption in ascribing all the quiescent luminosity to the heat that comes from inside of the star may not be a good estimate for this system, but here we are only interested in obtaining upper limits on $r$-mode amplitude.

\section{3. r-mode Spin-down}

To see whether or not these results are plausible and what fraction of the quiescent spin-down of these sources can be due to gravitational radiation from $r$-mode oscillations, we use our results for $\alpha$ from the "thermal equilibrium" condition and insert them into the right hand side of Equation (12a) to determine $\dot{\nu}$ in the absence of accretion $\left(N_{a c c}=0\right)$. The derived spin-down values for different NS models are given in Table 4 and are shown graphically in Figure 2 (vertical green lines). Comparing these results with the observed spin-down rates, which exist for IGR J00291, XTE 1751-305 and SAX J1808 (red diamonds in Figure 2), we find that in the case of $1.4 M_{\odot}$ (× symbols) and $2.0 M_{\odot}$ (square symbols) NSs, where there is no fast neutrino cooling present in the star, the $r$-mode spin-down can only provide about $1 \%$ of the observed spin-down rate, which means that other spin-down mechanisms such as magnetic-dipole radiation are responsible for spinning down a $1.4 M_{\odot}$ or $2.0 M_{\odot}$ hadronic star with no fast cooling process. For the two AMXPs with relatively "slow" spin frequencies, NGC 6440 and XTE J1814-338, we find $r$-mode spin-down limits that are more competitive with observed values. Indeed, our limit for NGC 6440 is comparable to the measured, quiescent spin-down rate for SAX J1808, and thus spin-down measurements for this source would be particularly interesting.

On the other hand, the $r$-mode amplitudes we obtain for the $2.21 M_{\odot}$ (circle symbols) hadronic model with direct Urca neutrino emission are only consistent with observations for SAX J1808 (and perhaps MXB 1659), as the inferred spin-down rates are either less than the observed rate for the source-in the case of SAX J1808-or similar to the other observed rates, as for MXB 1659. For the remaining sources considered here the $2.21 M_{\odot}$ limits are likely not consistent with the observations since such large amplitudes imply very large $r$-mode spin-down rates, and in the case of IGR J00291 and XTE J1814 they are in fact larger than the observed values. If the neutrino luminosity from these sources was indeed as large as estimated with our $2.21 M_{\odot}$ model, then in thermal equilibrium there must be a heat source that can supply it. Since the spin-down measurements for these sources indicate that $r$-mode heating (for this model) would be insufficient, several possibilities remain. First, there could be some additional source of heat other than $r$-mode dissipation that supplies the needed energy. However, we note that it would need to supply a substantial luminosity, as the direct Urca neutrino emission for this model outshines the photon luminosity by more than an order of magnitude, and we are not aware of any simple mechanisms that could provide the required luminosity. Second, the actual mass of these systems could be less than that of the model in question $\left(2.21 M_{\odot}\right)$. Indeed, if it could be demonstrated that $r$-mode dissipation were the only mechanism that could produce such a large luminosity then an upper limit on the mass would follow, and the limit would be the mass for which the neutrino plus photon luminosity matched the $r$-mode heating produced when the amplitude is large enough to produce a spindown rate equal to the observed quiescent rate or our theoretical value for a high mass NS model, whichever is smaller. This would be a conservative limit in the sense that it is likely that the $r$-mode torque does not account for all of the observed spin-down. Finally, our model assumptions, for example, the EOS and core composition, could be incorrect, with one possibility being the existence of exotic matter, such as kaon or pion condensates, or quark matter in the core which have smaller neutrino emissivities than nucleon direct Urca processes, or if the pairing gaps for ${ }^{3} P_{2}$ neutrons and ${ }^{1} S_{0}$ protons were larger than current theoretical values (this will be explained in more detail in the next paragraph). Interestingly, if the masses of these systems were known then one of the possibilities outlined above is precluded and then the observationally derived $r$-mode limits become sensitive to properties of the core, either the presence of exotic matter or perhaps additional heating physics. In this sense further spin-down measurements, and where possible, mass constraints could provide interesting new insights on the physics of dense NS matter.

Our theoretical treatment of neutrino emission processes in this study, namely modified and direct Urca, spans the plausible range between "slow" and "fast" neutrino cooling processes. Here we have considered NS models made of non-superfluid hadronic matter with the APR EOS, but more realistically it is likely that neutrons and protons will be in a superfluid phase inside NSs. Therefore, a natural question would be whether or not our conclusions will still hold in the presence of superfluidity. Considering the presence of superfluidity in these sources, assuming that they are still inside the unstable region for $r$-modes, could have two possible effects on their cooling. The first one is neutrino emission due to Cooper pair breaking and formation (PBF) just below the superfluid critical temperature (Page et al. 2004) and the second one is the suppression of direct and modified Urca neutrino cooling at temperatures below the critical temperature. Here we explain why our qualitative results, and our argument about setting upper bounds on the masses of these sources, are not changed by considering the effect of superfluidity on the neutrino cooling of these sources. In our low mass NS models $\left(M<2 M_{\odot}\right)$ where there is no fast cooling mechanism in the core, the thermal luminosity is much larger than the modified Urca neutrino luminosity, in fact by more than five orders of magnitude in all of the sources considered here except $4 \mathrm{U}$ 1608. Therefore, even if the temperature of these sources were just below the critical temperature of superfluidity, neutrino emission due to PBF, which is only about 10 times stronger than modified Urca neutrino emission, would still be much smaller than the thermal emission. Therefore, suppression of neutrino emission, or neutrino 
emission due to PBF is not important for our low mass NS models. What about high mass NSs where fast cooling can happen in the core? Since direct Urca neutrino emission is much stronger than neutrino emission due to PBF, if direct Urca is not suppressed by superfluidity, it will be the dominant cooling mechanism. Now the question is whether or not neutron and proton pairing can suppress direct Urca neutrino emission in the core. Current theoretical results for the pairing gaps in ${ }^{3} P_{2}$ neutron superfluid (Schwenk \& Friman 2004; Dong et al. 2013) and ${ }^{1} S_{0}$ proton superconducting phases (see for example Page et al. (2004); Yakovlev \& Pethick (2004)), which are relevant in the core of NSs, suggest that both of these pairing gaps are vanishingly small in the inner core, where the direct Urca process can operate. Therefore, superfluidity is unlikely to suppress fast cooling processes in the core of NSs and thus neglecting the effect of superfluidity does not change our qualitative results, assuming that $r$-modes are still unstable in the presence of superfluidity in these sources.

We also note that the density profile (density versus radius) for an NS made of hadronic matter with the APR EOS is almost flat at the center of the star, which means that as the mass of the star increases above $2 M_{\odot}$ (above which direct Urca processes can operate in the core of an NS made of hadronic matter with the APR EOS), there will be a sizable region in the core where direct Urca processes may happen, which can make it easier to obtain an upper limit on the mass of these sources using spin-down measurements.

With typical values of a few $\times 10^{-8}$, our derived amplitude upper limits suggest that for many LMXB NSs the $r$-modes are likely not excited to sufficient amplitudes to substantially affect their spin evolution. This begs the question of whether or not unstable, steadystate $r$-modes actually exist in these NSs. One possibility is that additional damping mechanisms, such as those perhaps associated with crust effects, such as the viscous friction at the crust-core boundary due to the coupling between core $r$-modes and crustal torsional modes (Levin \& Ushomirsky 2001), superfluid mutual friction (Ho et al. 2011) or the existence of exotic matter in the core of NSs (Alford et al. 2012a; Schwenzer 2012), are at work and modify the instability window so as to render these NSs stable to $r$-mode excitation. An interesting related question is whether the existence of $r$-modes at the amplitudes estimated here can be inferred directly from observations. Figure 2 shows both $r$-mode (dashed parallel lines) and magnetic-dipole (solid parallel lines) spin-down laws. The $r$-mode spin-down braking index, $n=7$, is steeper compared to that for magnetic spindown $(n=3)$, thus at high enough spin frequencies (well above a $\mathrm{kHz}$ ) one might expect that the $r$-mode torque would eventually become competitive with or dominate the magnetic dipole torque. However, as of yet there are no known NSs spinning fast enough for this effect to become dominant, and depending on the EOS the massshedding limit might be reached before the $r$-mode torque becomes competitive with the magnetic torque.

As discussed above, quiescent spin-down measurements have been typically attributed to the magnetic torque. For a number of sources considered here our $r$-mode amplitude limits support this presumption. Any spin-down contribution from an $r$-mode torque would be more easily identifiable if the magnetic field strengths of these NSs were constrained independently from the magnetic spin-down estimate. Moreover, identifying an $r$-mode spin-down would, in principle, be simpler for those NSs with the lowest magnetic torques, and thus field strengths. At present, the lowest inferred dipolar magnetic field strengths are $\approx 6 \times 10^{7} \mathrm{G}$. At this level the magnetic spin-down is of the order of $\dot{\nu} \approx 5 \times 10^{-17}$ $\mathrm{Hz} \mathrm{s}^{-1}$, which is comparable to our derived $r$-mode spindown limits for a number of sources considered here, assuming that their NSs are $<2 M_{\odot}$. In this regard, quiescent spin-down measurements for more of the sources considered here, in particular the AMXPs XTE J1814 and NGC 6440, would be extremely valuable.

\subsection{Gravitational Wave Amplitudes}

$r$-modes in NSs are one of the possible mechanisms for gravitational wave (GW) emission and they can be observationally interesting in newborn NSs and perhaps accreting NSs in LMXBs (Owen 2010). Continuous GW emission from $r$-modes is dominated by $l=m=2$ current quadrupole emission (Lindblom et al. 1998). The gravitational wave amplitude $h_{0}$ (strain tensor amplitude) is related to the $r$-mode amplitude $\alpha$ by the following equation (Owen 2010, 2009)

$$
h_{0}=\sqrt{\frac{8 \pi}{5}} \frac{G}{c^{5}} \frac{1}{r} \alpha \omega_{r}^{3} M R^{3} \tilde{J},
$$

where $r$ is the distance to the source, $M$ and $R$ are the mass and radius of the NS, $\tilde{J}$ is the dimensionless parameter defined by Equation (3) and $\omega_{r}$ is the frequency of the $r$-mode, which is related to the angular spin frequency of the star $\Omega$ (for the $m=l=2 r$-mode) by the following equation

$$
\omega_{r} \approx \frac{4}{3} \Omega .
$$

Using the upper limits on the $r$-mode amplitude of NSs in LMXBs derived above, we can obtain upper limits on the amplitude of the GWs emitted from these sources due to unstable $r$-modes. For the sources considered in this work, upper limits on the GW strain amplitude $h_{0}$ for the $1.4 M_{\odot}$ and $2.0 M_{\odot}$ NS models are in the range of $1.8 \times 10^{-29}$ to $4.9 \times 10^{-28}$ which is below the anticipated detectability threshold of Advanced LIGO Abbott et al. 2010; Watts et al. 2008). In the case of the $2.21 M_{\odot} \mathrm{NS}$ model, since the $r$-mode amplitudes are larger than those for the low mass NSs, we get larger values of $h_{0}$, but even in this case for most of the sources $h_{0}$ is still below the detectability threshold of Advanced LIGO. The highest values of $h_{0}$ for a massive star are obtained for NGC 6440 and Aql X-1 with an amplitude of the order of $8.5 \times 10^{-27}$ and $4 \mathrm{U} 1608-522$ with an amplitude of $1.59 \times 10^{-25}$. However, it should be noted that the large $r$-mode amplitudes in these sources, which would cause very large spin-down rates, may eventually be ruled out by future spin-down measurements. In this context it is important to restore the X-ray timing capability that was lost when $R X T E$ was decommissioned in January 2012. Missions planned or currently in development which could provide such a capability include India's ASTROSAT (Stewart|2012), ESA's Large Observatory for X-ray Timing (Feroci et al. 
2012), and NASA's Neutron Star Interior Composition Explorer (Gendreau et al. 2012).

The upper limits on GW amplitudes discussed here are related to the GW emission due to unstable $r$-modes but our results do not exclude the possibility of having larger GW amplitudes in LMXBs from other GW emission mechanisms such as NS mountains Ushomirsky et al. 2000; Haskell et al. 2006). It is worth mentioning that indirect upper limits on GW amplitude can be obtained for sources with observed spin-down rates, $\dot{\nu}$, by assuming that all of the observed spin-down is due to $\mathrm{GW}$ emission (Owen 2010),

$$
h_{0}^{s d}=\frac{1}{r} \sqrt{\frac{45 G I \dot{P}}{8 c^{3} P}}
$$

where $P=\frac{2 \pi}{\Omega}$ is the observed pulse period and $\left|\frac{\dot{P}}{P}\right|=\left|\frac{\dot{\nu}}{\nu}\right|$. Using this equation for the three sources with measured $\dot{\nu}$ and different NS models (i.e. different masses and radii) we obtain $h_{0}^{s d}$ values that range from $4.14 \times 10^{-28}$ to $6.53 \times 10^{-28}$.

\section{CONCLUSIONS}

In this paper we have presented upper limits on $r$-mode amplitudes in LMXB NSs using their observed quiescent luminosities, temperatures and spin-down rates. We calculated results for NS models constructed with the APR EOS (normal hadronic matter) with masses of 1.4, 2 and $2.21 M_{\odot}$, where our highest mass model $\left(2.21 M_{\odot}\right)$ can support enhanced, direct Urca neutrino emission in the core. We have used two different methods to calculate $r$-mode amplitudes. The first is based on the assumption that in an outburst-quiescence cycle all the spin-up torque due to accretion during the outburst is balanced by the $r$-mode spin-down torque due to gravitational radiation. This method gives amplitudes in the range of $\approx 1-3 \times 10^{-7}$ for the sources with measured spin-up rates. Since in reality there are other sources of spindown such as magnetic-dipole radiation that may be the dominant spin-down source, we use another method for computing the $r$-mode amplitude that does not ascribe all of the spin-down of the star to gravitational radiation and therefore gives tighter bounds on the amplitudes. This second method is based on the assumption that in a thermal steady-state some fraction of the heat that is generated in the star due to $r$-mode dissipation will be lost from the star by neutrino emission and the rest will be radiated at the surface. This assumes that all of the heat emitted from the surface of the star during quiescence is due to the $r$-mode dissipation inside the star, and thus provides an upper bound on the $r$-mode amplitude. We have computed core temperatures as well as neutrino and thermal (photon) luminosities for LMXB sources using measurements of the quiescent luminosities and surface temperatures and showed that at temperatures relevant for LMXB neutron stars, when there is no enhanced cooling mechanism, the cooling of the star is dominated by photon emission from the surface (for 1.4 and $2.0 M_{\odot} \mathrm{NS}$ models), but in a massive star where direct Urca neutrino emission is allowed, the cooling is dominated by neutrino emission (for $T_{\text {eff }} \gtrsim 34$ $\mathrm{eV})$. For the lower mass NS models (1.4 and $\left.2 M_{\odot}\right)$ we find dimensionless $r$-mode amplitudes in the range from about $1 \times 10^{-8}$ to $1.5 \times 10^{-6}$. We note that none of the saturation mechanisms proposed so far can saturate $r$-modes at these low amplitudes. Alternatively, the enhanced dissipation that would result from the existence of exotic matter in NS interiors could shift the instability window such that the LMXBs are perhaps stable to $r$-mode excitation (Alford et al. 2012a; Schwenzer 2012).

For the AMXP sources with known quiescent spindown rates these limits suggest that $\lesssim 1 \%$ of the observed rate can be due to an unstable $r$-mode. Interestingly, the AMXP with the highest amplitude limit, NGC 6440, could have an $r$-mode spin-down rate comparable to the observed, quiescent rate for SAX J1808. Thus, quiescent spin-down measurements for this source would be particularly interesting. Having enhanced, direct Urca neutrino emission in the core of our highest mass model $\left(2.21 M_{\odot}\right)$ means that the dissipated heat in the star can be larger and therefore it can have higher $r$-mode amplitudes. Indeed, the inferred $r$-mode spin-down rates at these higher amplitudes are inconsistent with the observed spin-down rates for some of the LMXB sources, such as IGR J00291 and XTE J1751-305. If $r$-mode dissipation were the only mechanism available to produce this high luminosity, then this could be used to put an upper limit on the masses of these sources if they were made of hadronic matter. Alternatively, it could be used to probe the existence of exotic matter in them if the NS mass in these systems were known. In this way, future spin-down and NS mass measurements for the LMXB systems considered here, as well as for yet to be discovered systems, could open a new window on dense matter in NS interiors. For this as well as other reasons, we regard the re-establishment of a sensitive X-ray timing capability as vital to the use of NSs as natural laboratories for the study of dense matter. Using the results for $r$ mode amplitudes, the upper limits on gravitational wave amplitude due to $r$-modes have been computed. The upper limits on the GW strain amplitude $h_{0}$ for the $1.4 M_{\odot}$ and $2.0 M_{\odot}$ NS models are in the range of $1.8 \times 10^{-29}$ to $4.9 \times 10^{-28}$ which is below the anticipated detectability threshold of Advanced LIGO. In the case of the $2.21 M_{\odot}$ NS model, we obtain larger values for $h_{0}$, but even in this case for most of the sources considered in this work, $h_{0}$ is still below the detectability threshold of Advanced LIGO. Gravitational waves due to other mechanisms such as NS mountains may have larger amplitudes in these systems.

SM thanks Mark Alford, Andrew Cumming, Cole Miller and Kai Schwenzer for helpful discussions. TS acknowledges NASA's support for high energy astrophysics. SM acknowledges the support of the U.S. Department of Energy through grant No. DEFG02- 93ER40762.

\section{REFERENCES}


Alford, M. G., Mahmoodifar, S., \& Schwenzer, K. 2010, J. Phys., G37, 125202

Alford, M., Mahmoodifar, S., \& Schwenzer, K. 2012a, Phys.Rev., D85, 024007

—. 2012b, AIP Conf.Proc., 1492, 257

-. 2012c, Phys.Rev., D85, 044051

Alford, M. G., \& Schwenzer, K. 2012, arXiv:1210.6091

Andersson, N. 1998, Astrophys. J., 502, 708

Andersson, N., Kokkotas, K. D., \& Stergioulas, N. 1999, Astrophys.J., 516, 307

Arras, P., Flanagan, E. E., Morsink, S. M., et al. 2003, Astrophys.J., 591, 1129

Bildsten, L., \& Ushomirsky, G. 2000, Astrophys. J. Lett., 529, L33

Bondarescu, R., Teukolsky, S. A., \& Wasserman, I. 2007, Phys. Rev. D, 76, 064019

- 2009, Phys. Rev. D, 79, 104003

Brown, E. F., Bildsten, L., \& Rutledge, R. E. 1998, arXiv:astro-ph/9807179

Brown, E. F., \& Cumming, A. 2009, Astrophys.J., 698, 1020

Brown, E. F., \& Ushomirsky, G. 2000, ApJ, 536, 915

Chakrabarty, D., Morgan, E. H., Muno, M. P., et al. 2003, Nature, 424, 42

Degenaar, N., Patruno, A., \& Wijnands, R. 2012, Astrophys.J., 756,148

Dong, J., Lombardo, U., \& Zuo, W. 2013, Phys.Rev., C87, 062801

Feroci, M., den Herder, J. W., Bozzo, E., et al. 2012, in Society of Photo-Optical Instrumentation Engineers (SPIE) Conference Series, Vol. 8443, Society of Photo-Optical Instrumentation Engineers (SPIE) Conference Series

Friedman, J. L., \& Morsink, S. M. 1998, Astrophys. J., 502, 714

Friedman, J. L., \& Schutz, B. F. 1978, Astrophys. J., 222, 281

Gendreau, K. C., Arzoumanian, Z., \& Okajima, T. 2012, in Society of Photo-Optical Instrumentation Engineers (SPIE) Conference Series, Vol. 8443, Society of Photo-Optical Instrumentation Engineers (SPIE) Conference Series

Gupta, S., Brown, E. F., Schatz, H., Möller, P., \& Kratz, K.-L. 2007, ApJ, 662, 1188

Haensel, P., \& Zdunik, J. L. 2003, A\&A, 404, L33

Hartman, J. M., Patruno, A., Chakrabarty, D., et al. 2009, ApJ, 702,1673

Haskell, B., Degenaar, N., \& Ho, W. C. G. 2012, MNRAS, 424, 93

Haskell, B., Jones, D., \& Andersson, N. 2006, Mon.Not.Roy.Astron.Soc., 373, 1423

Haskell, B., \& Patruno, A. 2011, ApJ, 738, L14

Hebeler, K., Lattimer, J., Pethick, C., \& Schwenk, A. 2013, arXiv:1303.4662

Heinke, C., Jonker, P., Wijnands, R., Deloye, C., \& Taam, R. 2009, Astrophys.J., 691, 1035
Heinke, C. O., Jonker, P., Wijnands, R., \& Taam, R. 2007, Astrophys.J., 660, 1424

Hessels, J. W. T., Ransom, S. M., Stairs, I. H., et al. 2006, Science, 311, 1901

Ho, W. C., Andersson, N., \& Haskell, B. 2011, Phys.Rev.Lett., 107,101101

Ho, W. C., \& Lai, D. 2000, Astrophys.J., 543, 386

Lattimer, J. M. 2012, Ann.Rev.Nucl.Part.Sci., 62, 485

Levin, Y., \& Ushomirsky, G. 2001, Mon.Not.Roy.Astron.Soc., 324,917

Lindblom, L., Owen, B. J., \& Morsink, S. M. 1998, Phys. Rev. Lett., 80,4843

Manchester, R. N., Hobbs, G. B., Teoh, A., \& Hobbs, M. 2005, Astron.J., 129, 1993

Owen, B. J. 2009, Class.Quant.Grav., 26, 204014

—. 2010, Phys.Rev., D82, 104002

Owen, B. J., et al. 1998, Phys. Rev., D58, 084020

Page, D., Lattimer, J. M., Prakash, M., \& Steiner, A. W. 2004, Astrophys.J.Suppl., 155, 623

Patruno, A. 2010, Astrophys.J., 722, 909

Patruno, A., Bult, P., Gopakumar, A., et al. 2011, arXiv:1111.6967

Patruno, A., Haskell, B., \& D'Angelo, C. 2012, ApJ, 746, 9

Patruno, A., \& Watts, A. 2012, arXiv:1206.2727

Potekhin, A., Chabrier, G., \& Yakovlev, D. 1997, arXiv:astro-ph/9706148

Rezzolla, L., Lamb, F. K., Marković, D., \& Shapiro, S. L. 2001, Phys. Rev. D, 64, 104014

Rezzolla, L., Lamb, F. K., \& Shapiro, S. L. 2000, ApJ, 531, L139

Schwenk, A., \& Friman, B. 2004, Phys.Rev.Lett., 92, 082501

Schwenzer, K. 2012, arXiv: 1212.5242

Stewart, G. 2012, in Half a Century of X-Ray Astronomy,

Proceedings of the conference held 17-21 September, 2012 on Mykonos Island, Greece, id.95

Tomsick, J. A., Gelino, D. M., Halpern, J. P., \& Kaaret, P. 2004, Astrophys.J., 610, 933

Ushomirsky, G., Cutler, C., \& Bildsten, L. 2000,

Mon.Not.Roy.Astron.Soc., 319, 902

Watts, A., Krishnan, B., Bildsten, L., \& Schutz, B. F. 2008, Mon.Not.Roy.Astron.Soc., 389, 839

White, N. E., \& Zhang, W. 1997, ApJ, 490, L87

Wu, Y., Matzner, C. D., \& Arras, P. 2001, Astrophys.J., 549, 1011

Yakovlev, D. G., \& Pethick, C. 2004, Ann.Rev.Astron.Astrophys., 42,169 